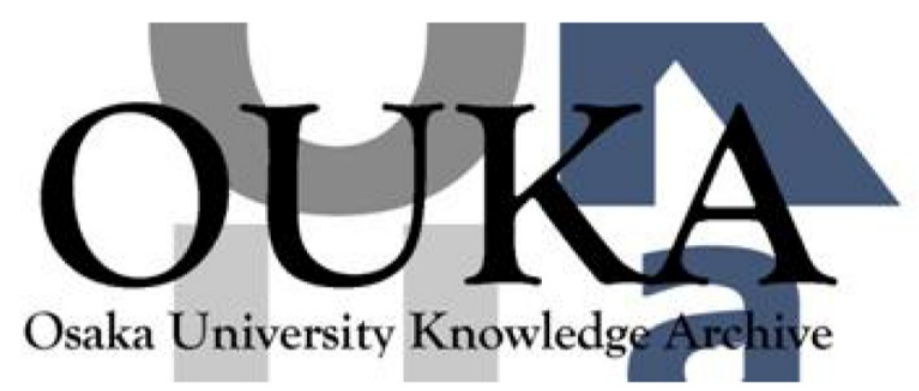

\begin{tabular}{|c|l|}
\hline Title & $\begin{array}{l}\text { Analytical Expression Based Design of a Low- } \\
\text { Voltage FD-SOI CMOS Low-Noise Amplifier }\end{array}$ \\
\hline Author(s) & $\begin{array}{l}\text { Kihara, Takao; Kim, Guechol; Goto, Masaru et } \\
\text { al. }\end{array}$ \\
\hline Citation & $\begin{array}{l}\text { IEICE TRANSACTIONS on Fundamentals of } \\
\text { Electronics, Commun icat ions and Computer } \\
\text { Sciences. E90-A(2) p.317-p.325 }\end{array}$ \\
\hline Issue Date & $2007-02$ \\
\hline oaire:version & VoR \\
\hline URL & https://hdl. handle. net/11094/51688 \\
\hline rights & copyright $\odot 2007$ IEICE \\
\hline Note & \\
\hline
\end{tabular}

Osaka University Knowledge Archive : OUKA

https://ir. Library. osaka-u. ac. jp/

Osaka University 


\section{Analytical Expression Based Design of a Low-Voltage FD-SOI CMOS Low-Noise Amplifier}

Takao KIHARA $^{\dagger a)}$, Guechol KIM ${ }^{\dagger}$, Masaru GOTO ${ }^{\dagger}$, Keiji NAKAMURA ${ }^{\dagger}$, Nonmembers, $^{\text {, }}$ Yoshiyuki SHIMIZU ${ }^{\dagger}$, Student Member, Toshimasa MATSUOKA ${ }^{\dagger}$, and Kenji TANIGUCHI ${ }^{\dagger}$, Members

\begin{abstract}
SUMMARY We propose a design methodology of a low-voltage CMOS low-noise amplifier (LNA) consisting of a common-source and a common-gate stages. We first derive equations of power gain, noise figure (NF) and input third-order intercept point $\left(\mathrm{IIP}_{3}\right)$ of the two-stage LNA. A design methodology of the LNA is presented by using graphs based on analytical equations. A 1-V 5.4-GHz LNA was implemented in $0.15-\mu \mathrm{m}$ fullydepleted silicon-on-insulator (FD-SOI) CMOS technology. Measurement results show a power gain of $23 \mathrm{~dB}$, NF of $1.7 \mathrm{~dB}$ and $\mathrm{IIP}_{3}$ of $-6.1 \mathrm{dBm}$ with a power consumption of $8.3 \mathrm{~mW}$. These measured results are consistent with calculated results, which ensures the validity of the derived equations and the proposed design methodology.

key words: low-noise amplifier (LNA), FD-SOI CMOS, noise, linearity, low-voltage
\end{abstract}

\section{Introduction}

Recently, the supply voltage of integrated circuits has been reduced because of MOSFET miniaturization and the increasing demand for low power consumption. It is, however, difficult to realize a radio-frequency $(\mathrm{RF})$ circuit with desired performance at low supply voltage. Promising solutions are two-folds; to develop circuit topologies suitable for low supply voltage and to design circuits in a new process such as the silicon-on-insulator (SOI) process [1], [2].

The first building block of a wireless receiver is the low-noise amplifier (LNA) which amplifies small signals received by an antenna. It has to keep the internal noise as low as possible and to have sufficient high power gain. LNA also requires good linearity to reduce distortion [3], [4]. Considering both low noise and high linearity, it is difficult to design a cascode LNA [5], [6] operating below 1-V supply voltage, although the design methodology of a $2.5-\mathrm{V}$ cascode LNA [7] has been reported.

We investigate the structure of a low-voltage LNA and proposes a new design methodology of the LNA taking power gain, noise and linearity into account. We implement a $1-\mathrm{V}$ 5.4-GHz LNA in $0.15-\mu \mathrm{m}$ fully-depleted SOI (FDSOI) CMOS technology and verify both its performance and our design methodology.

This paper is organized as follows. Section 2 describes

Manuscript received June 19, 2006.

Manuscript revised September 7, 2006.

Final manuscript received October 5, 2006.

†The authors are with the Department of Electrical, Electronics and Information Engineering, Graduate School of Engineering, Osaka University, Suita-shi, 565-0871 Japan.

a)E-mail: kihara@si.eei.eng.osaka-u.ac.jp DOI: 10.1093/ietfec/e90-a.2.317 the circuit topology of a low-voltage LNA. Section 3 shows the small-signal equivalent circuit of the LNA and Sect. 4 derives equations of power gain, noise figure (NF) and input third-order intercept point $\left(\mathrm{IIP}_{3}\right)$ of the LNA. Section 5 presents the design methodology. Section 6 shows the measurement results of the fabricated LNA.

\section{Two-Stage LNA}

Figure 1 shows a conventional cascode LNA with inductive source degeneration [5], [6] which is widely used as a CMOS LNA. The overdrive voltage of the transistor $M_{i}$ is expressed as

$$
V_{o d i}=V_{g s i}-V_{t h i}
$$

where $V_{g s i}$ and $V_{t h i}$ are the gate-source voltage and threshold voltage of $M_{i}$, respectively. It is difficult to use this cascode LNA at low supply voltage because the supply voltage must exceed two overdrive voltages, $V_{o d 1}+V_{o d 2}$.

In this study, we adopt a two-stage LNA consisting of a common-source and a common-gate stages [8] as shown in Fig. 2. The ac-coupling capacitor $C_{c}$ is placed between the input and output stages. The two internal LC tanks $L_{1} / C_{1}$ and $L_{2} / C_{2}$ are chosen to resonate at the operation frequency. The transistor $M_{2}$ improves reverse isolation and reduces the Miller effect. The minimum supply voltage of the LNA is only $V_{o d 1}$, so that the LNA operates at lower supply voltage than the conventional cascode LNA.

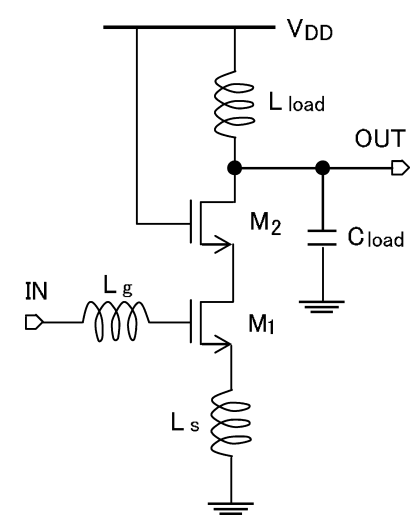

Fig. 1 Schematic of conventional cascode LNA. 


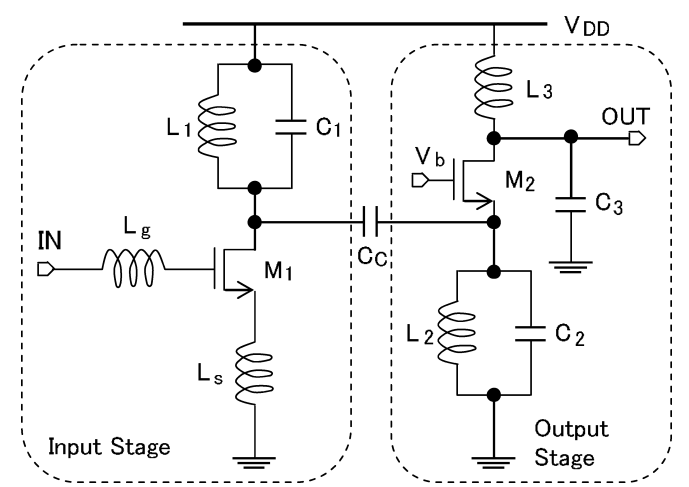

Fig. 2 Schematic of two-stage LNA studied.

\section{Small-Signal Equivalent Circuit of the LNA}

Figure 3 shows the input stage of the two-stage LNA, where $v_{s}$ and $R_{s}$ are a signal source voltage and signal source impedance, respectively. For more precise derivations than those reported in [7], we take account of a parasitic capacitance $C_{p}$ originating from both the input pad capacitance and gate-drain capacitance of $M_{1}, C_{g d 1}$ [4]. $R_{e q}+j \omega_{0} L_{e q}$ denotes the equivalent source impedance looking into the left hand side of the reference plane 2 , which is given by

$$
\begin{aligned}
R_{e q} & =\frac{R_{s}}{\omega_{0}^{2} C_{p}^{2} R_{s}^{2}+\left(1-\omega_{0}^{2} C_{p} L_{g}\right)^{2}}, \\
L_{e q} & =\frac{L_{g}-C_{p}\left(\omega_{0}^{2} L_{g}^{2}+R_{s}^{2}\right)}{\omega_{0}^{2} C_{p}^{2} R_{s}^{2}+\left(1-\omega_{0}^{2} C_{p} L_{g}\right)^{2}} .
\end{aligned}
$$

Figure 4 shows the small-signal equivalent circuit of the input stage with the equivalent signal source impedance. Note that an SOI MOSFET has no body effect in the equivalent circuit. The equivalent signal source voltage $v_{s, e q}$ is given by

$$
v_{s, e q}=\frac{v_{s}}{1-\omega_{0}^{2} C_{p} L_{g}+j \omega_{0} C_{p} R_{s}} .
$$

The effective gate-source capacitance of $M_{1}, C_{g s 1, e f f}$, effective inductance, $L_{s, e f f}$, and non-quasi-static (NQS) resistance, $r_{n q s, e f f}$, are expressed as follows [4]:

$$
\begin{aligned}
& C_{g s 1, e f f}=\left(1+M \alpha_{g d}\right) C_{g s 1}, \\
& L_{s, e f f}=\frac{L_{s}}{1+M \alpha_{g d}}, \\
& r_{n q s, e f f}=\frac{r_{n q s}}{1+M \alpha_{g d}}, \\
& M=g_{m 1} \times\left(\frac{1}{g_{m 2}} / / R_{I}\right)=\frac{g_{m 1} R_{I}}{1+g_{m 2} R_{I}}, \\
& \alpha_{g d}=\frac{C_{g d}}{C_{g s}}
\end{aligned}
$$

where $g_{m i}$ is the transconductance of $M_{i}, M$ the Miller factor and $R_{I}$ the parallel resistance of $R_{L_{1}, p}$ and $R_{L_{2}, p} . R_{L_{i}, p}$ is the

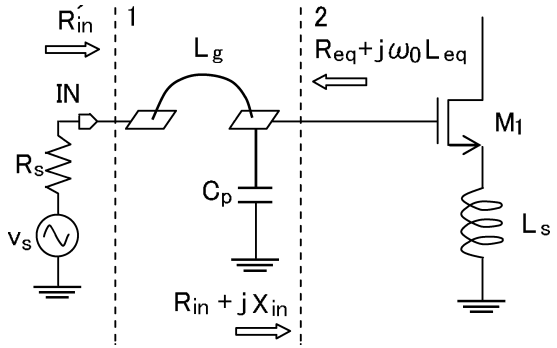

Fig. 3 Input stage of LNA.

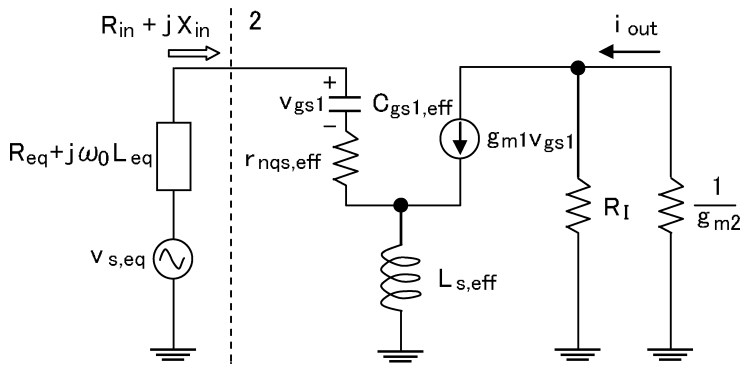

Fig. 4 Small-signal equivalent circuit of input stage.

equivalent resistance of the internal and load LC tanks consisting $L_{i}$ and $C_{i}(i=1,2,3)$ at a resonant frequency, which is given by

$$
R_{L_{i}, p} \simeq \frac{\omega_{0}^{2} L_{i}^{2}}{R_{L_{i}, s}}
$$

where $R_{L_{i}, s}$ is the series resistance of $L_{i}$. From Eqs. (5)-(9), the real and imaginary parts of the input impedance looking into the right hand side of the reference plane 2 are given by

$$
\begin{aligned}
& R_{\text {in }}=r_{n q s, e f f}+\omega_{T 1} L_{s, e f f}, \\
& X_{\text {in }}=\omega_{0} L_{s, e f f}-\frac{1}{\omega_{0} C_{g s 1, e f f}}
\end{aligned}
$$

where $\omega_{T i}=g_{m i} / C_{g s i} . R_{i n}^{\prime}$ denotes the input impedance of the LNA looking into the right hand side of the reference plane 1 , which is given by

$$
R_{\text {in }}^{\prime}=\frac{R_{\text {in }}\left(1-\omega_{0}^{2} L_{g} C_{p}\right)+j\left(\omega_{0} L_{g}+X_{\text {in }}-\omega_{0}^{2} L_{g} C_{p} X_{i n}\right)}{1-\omega_{0} C_{p} X_{i n}+j \omega_{0} C_{p} R_{\text {in }}} .
$$

From Fig. 4, the impedance matching condition is given by

$$
R_{\text {in }}^{\prime}=R_{s}
$$

or

$$
\begin{aligned}
& R_{e q}=R_{i n}, \\
& \omega_{0} L_{e q}=-X_{i n} .
\end{aligned}
$$

\section{Power Gain, NF and $\mathrm{IIP}_{3}$ of Two-Stage LNA}

For our design optimization, we derive expressions of power gain, $\mathrm{NF}$ and $\mathrm{IIP}_{3}$ of the two-stage LNA. 


\subsection{Power Gain}

The output power of the LNA is given by the output load $R_{L_{3}, p}$ and the current injected in the load $i_{\text {out }}$ as

$$
P_{\text {out }}=\left|i_{\text {out }}\right|^{2} R_{L_{3}, p}
$$

where $R_{L_{3}, p}$ is given by Eq. (10). Based on the equivalent circuit shown in Fig. $4,\left|i_{\text {out }}\right|^{2}$ is given by

$$
\left|i_{\text {out }}\right|^{2}=\frac{4 P_{a v} R_{e q}}{\left(R_{e q}+R_{\text {in }}\right)^{2}}\left(\frac{\omega_{T 1}}{\omega_{0}}\right)^{2}\left(\frac{R_{I}}{R_{I}+1 / g_{m 2}}\right)^{2}
$$

where $P_{a v}$ is the available power of the source.

$$
P_{a v}=\frac{\left|v_{s, e q}\right|^{2}}{4 R_{e q}}=\frac{\left|v_{s}\right|^{2}}{4 R_{s}} .
$$

From Eqs. (17)-(19), the power gain of the two-stage LNA is derived as

$$
G_{t}=\frac{4 R_{e q} R_{L_{3}, p}}{\left(R_{e q}+R_{i n}\right)^{2}}\left(\frac{\omega_{T 1}}{\omega_{0}}\right)^{2}\left(\frac{R_{I}}{R_{I}+1 / g_{m 2}}\right)^{2} .
$$

\section{$4.2 \mathrm{NF}$}

Figure 5 shows the noise equivalent circuit of input stage of the LNA. The noise voltage, $v_{n s, e q}$, the drain noise and the induced-gate noise currents, $i_{n d i}$ and $i_{n g i}$, of $M_{i}(i=1,2)$ are expressed as

$$
\begin{aligned}
& \overline{\left|v_{n s, e q}\right|^{2}}=4 k_{B} T R_{e q} \Delta f, \\
& \overline{\left|i_{n d i}\right|^{2}}=4 k_{B} T \frac{\gamma_{i}}{\alpha_{i}} g_{m i} \Delta f, \\
& \overline{\left|i_{n g i}\right|^{2}}=4 k_{B} T \delta_{i} \frac{\left(\omega C_{g s i}\right)^{2}}{\kappa_{i} g_{d 0 i}} \Delta f
\end{aligned}
$$

where $\alpha_{i}=g_{m i} / g_{d 0 i}$ and $g_{d 0 i}$ is the zero-bias drain conductance of $M_{i}, \gamma_{i}, \delta_{i}$ and $\kappa_{i}$ are the noise parameters of $M_{i}$ [5], [6].

The output noise shown in Fig. 5 is calculated from Eq. (21) as

$$
\overline{\left|i_{o, s, e q}\right|^{2}}=\frac{4 k_{B} T R_{e q} \omega_{T 1}^{2} \Delta f}{\omega_{0}^{2}\left(1+\alpha_{g d} M\right)^{2}\left(R_{e q}+R_{i n}\right)^{2}\left(1+1 / g_{m 2} R_{I}\right)^{2}} .
$$

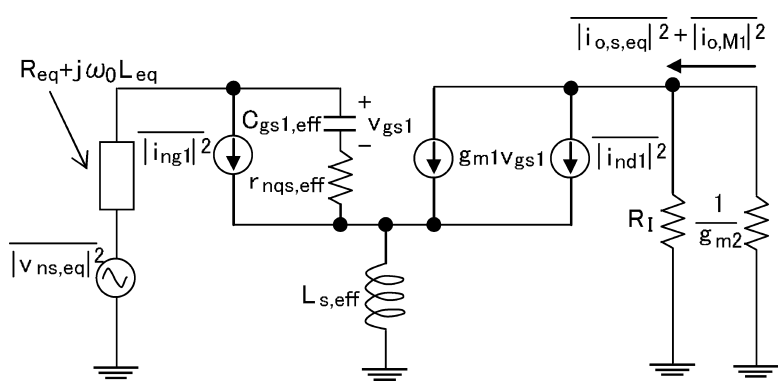

Fig. 5 Noise equivalent circuit of input stage.
From Eqs. (22) and (23), the equation of the output noise due to $M_{1}$ shown in Fig. 5 is derived as

$$
\begin{gathered}
\overline{\left|i_{o, M_{1}}\right|^{2}}=\frac{4 k_{B} T \gamma_{1} \chi_{1} g_{m 1}\left(R_{e q}+r_{n q s, e f f}\right)^{2} \Delta f}{\alpha_{1}\left(R_{e q}+R_{\text {in }}\right)^{2}\left(1+1 / g_{m 2} R_{I}\right)^{2}}, \\
\chi_{1}=1-\frac{2|c| \alpha_{1} R_{e q}}{\left(1+\alpha_{g d} M\right)\left(R_{e q}+r_{n q s, e f f}\right)} \sqrt{\frac{\delta_{1}}{\kappa_{1} \gamma_{1}}} \\
+\frac{\delta_{1} \alpha_{1}^{2}}{\kappa_{1} \gamma_{1}} \frac{R_{e q}^{2}+1 / \omega_{0}^{2} C_{g s 1, e f f}^{2}}{\left(1+\alpha_{g d} M\right)^{2}\left(R_{e q}+r_{n q s, e f f}\right)^{2}}
\end{gathered}
$$

where $c \simeq j 0.395$ is the correlation coefficient between the induced-gate and drain noise currents [5], [6]. The detailed derivations are summarized in the Appendix.

Figure 6 shows the noise equivalent circuit of the common-gate stage of the LNA. The noise current $i_{n R_{L_{i}}}$ originating from the internal or load LC tank is given by

$$
\overline{\left|i_{n R_{L_{i}}}\right|^{2}}=\frac{4 k_{B} T \Delta f}{R_{L_{i}, p}} .
$$

The detailed derivations of the output noise due to $M_{2}$ are also summarized in the Appendix. The output noise originating from $M_{2}$ is given by

$$
\begin{aligned}
& \overline{\left|i_{o, M_{2}}\right|^{2}}=4 k_{B} T \frac{\gamma_{2}}{\alpha_{2}} \chi_{2} g_{m 2} \Delta f, \\
& \chi_{2}=\frac{1}{\left(1+g_{m 2} R_{I}\right)^{2}}+\frac{1}{\left(1+1 / g_{m 2} R_{I}\right)^{2}}\left(\frac{\omega_{0}}{\omega_{T 2}}\right)^{2} \frac{\alpha_{2}^{2} \delta_{2}}{\kappa_{2} \gamma_{2}} .
\end{aligned}
$$

The output noises due to the internal and load LC tanks shown in Fig. 6 are derived from Eq. (27) as

$$
\begin{aligned}
& \overline{\left|i_{o, R_{I}}\right|^{2}}=\frac{4 k_{B} T \Delta f}{\left(1+1 / g_{m 2} R_{I}\right)^{2} R_{I}}, \\
& \overline{\left|i_{o, R_{L_{3}}}\right|^{2}}=\overline{\left|i_{n R_{L_{3}}}\right|^{2}}=\frac{4 k_{B} T \Delta f}{R_{L_{3, p}}} .
\end{aligned}
$$

Therefore, NF of the two-stage LNA results in

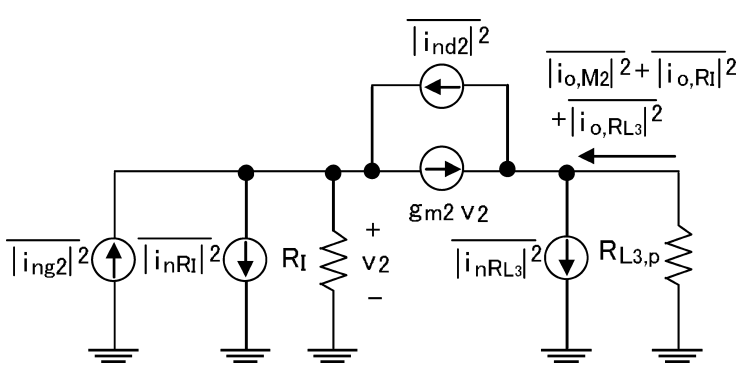

Fig. 6 Noise quivalent circuit of common-gate stage. 


$$
\begin{aligned}
& N F=\frac{\overline{\left|i_{o, s, e d}\right|^{2}}+\overline{\left|i_{o, M}\right|^{2}}+\overline{\left|i_{o, M_{2}}\right|^{2}}+\overline{\left|i_{o, R}\right|^{2}}+\overline{\left|i_{o, R_{L}}\right|^{2}}}{\overline{\left|i_{o, s, e d}\right|^{2}}} \\
& =1+\frac{\gamma_{1} \chi_{1}}{\alpha_{1}}\left(\frac{\omega_{0}}{\omega_{T 1}}\right)^{2} \frac{g_{m 1}\left(1+\alpha_{g d} M\right)^{2}\left(R_{e q}+r_{n q s, e f f}\right)^{2}}{R_{e q}} \\
& +\left(\frac{\omega_{0}}{\omega_{T 1}}\right)^{2} \frac{\left(1+\alpha_{g d} M\right)^{2}\left(R_{e q}+R_{i n}\right)^{2}}{R_{e q}}\left(1+\frac{1}{g_{m 2} R_{I}}\right)^{2} \\
& \\
& \times\left[\frac{\gamma_{2} \chi_{2} g_{m 2}}{\alpha_{2}}+\frac{1}{\left(1+1 / g_{m 2} R_{I}\right)^{2} R_{I}}+\frac{1}{R_{L_{3, p}}}\right] .
\end{aligned}
$$

\section{$4.3 \quad \mathrm{IIP}_{3}$}

The output of a non-linear small-signal amplifier $y(t)$ is approximately expressed as

$$
y(t) \approx a_{1} x(t)+a_{2} x^{2}(t)+a_{3} x^{3}(t)
$$

where $x(t)$ is an input signal. The $\mathrm{IIP}_{3}$ of the amplifier in the expression of voltage amplitude is given by [9]

$$
A_{I I P_{3}}=\sqrt{\frac{4}{3}\left|\frac{a_{1}}{a_{3}}\right|} .
$$

$\mathrm{IIP}_{3}$ usually expressed as the available power of signal source is given by

$$
I I P_{3}=\frac{A_{I I P_{3}}^{2}}{8 R_{s}} .
$$

The overall $A_{I I P}^{2}$ of the LNA consisting of the input and the output stages (Fig. 2) is given by [9]

$$
\frac{1}{A_{L N A, I I P_{3}}^{2}} \simeq \frac{1}{A_{1, I I P_{3}}^{2}}+\frac{A_{v 1}^{2}}{A_{2, I I P_{3}}^{2}}
$$

where $A_{1, I I P_{3}}$ and $A_{2, I I P_{3}}$ represent the $\mathrm{IIP}_{3}$ of the input and output stages in the expression of voltage amplitude, respectively. $A_{v 1}$ is the voltage gain of the input stage, which is given by

$$
A_{v 1}=\left|\frac{g_{m 1}\left(\frac{1}{g_{m 2}} / / R_{I}\right)}{j \omega_{0} C_{g s 1, e f f}\left(R_{e q}+R_{i n}\right)\left(1-\omega_{0}^{2} C_{p} L_{g}+j \omega_{0} C_{p} R_{s}\right)}\right| .
$$

The drain current of MOSFET at each stage is expressed as [10]

$$
I_{d i}=\frac{1}{2} \mu_{0} C_{o x} \frac{W_{i}}{L} \frac{V_{o d i}^{2}}{1+\Theta V_{o d i}} \frac{1}{1-\lambda V_{d s i}}
$$

where $W_{i}$ and $L$ are the gate width and length of $M_{i}, \lambda$ is the channel-length modulation coefficient, $\Theta=\mu_{0} /\left(2 v_{s a t} L\right)+$ $\theta, v_{\text {sat }}$ the saturation velocity of the carrier, $\mu_{0}$ the carrier mobility under low electric field and $\theta$ the mobility reduction parameter. From Eq. (38), for a signal $v_{s}(t)$ applied to the LNA, the output current of each stage is derived as

$$
\begin{aligned}
I_{d i}(t) & =\frac{c_{0}\left(c_{1}+c^{\prime} v_{i}(t)\right)^{2}}{c_{2}+c_{3} v_{i}(t)+c^{\prime 2} c_{4} v_{i}^{2}(t)}(i=1,2), \\
c^{\prime}= & \frac{1}{j \omega_{0} C_{g s 1, e f f}\left(R_{e q}+R_{i n}\right)} \\
& \times \frac{1}{1-\omega_{0}^{2} L_{g} C_{p}+j \omega_{0} C_{p} R_{s}}(i=1), \\
& -1(i=2), \\
c_{0}= & \frac{1}{2} \mu_{0} C_{o x} \frac{W_{i}}{L}, \\
c_{1}= & V_{o d i}, \\
c_{2}= & \left(1+\Theta V_{o d i}\right)\left(1-\lambda V_{d s i}\right), \\
c_{3}= & \left(1+\Theta V_{o d i}\right) \lambda A_{v i}+c^{\prime}\left(1-\lambda V_{d s i}\right) \Theta, \\
c_{4}= & \Theta \lambda A_{v i}
\end{aligned}
$$

where $v_{1}(t)=v_{s}(t)$ and $v_{2}(t)=-A_{v 1} v_{s}(t)$. In the derivation of Eqs. (39)-(46), the channel-length modulation in $M_{1}$ due to the variation of the source voltage is neglected. Substituting Taylor expansion coefficients of Eq. (39), $a_{1}$ and $a_{3}$, into Eq. (34), $A_{I I P_{3}}^{2}$ of each stage can be derived as

$$
A_{i, I I P_{3}}^{2}=\frac{4}{3}\left|\frac{c_{1} c_{2}^{2}\left(c_{1} c_{3}-2 c^{\prime} c_{2}\right)}{\left(-c^{\prime} c_{2}+c_{1} c_{3}\right)\left[\left(-c^{\prime} c_{2}+c_{1} c_{3}\right) c_{3}-2 c^{\prime} c_{1} c_{2} c_{4}\right]}\right| .
$$

The overall $\mathrm{IIP}_{3}$ of the LNA can be given by Eqs. (35), (36) and (47).

\section{Design of the Two-Stage LNA}

The transconductance of $M_{i}$ is a derivative of Eq. (38), which is given by

$$
g_{m i}=\frac{1}{2} \mu_{0} C_{o x} \frac{W_{i}}{L} \frac{V_{o d i}\left(2+\Theta V_{o d i}\right)}{\left(1+\Theta V_{o d i}\right)^{2}} \frac{1}{1-\lambda V_{d s i}} .
$$

The power gain, $\mathrm{NF}$ and $\mathrm{IIP}_{3}$ are expressed as a function of $V_{\text {odi }}$ by using Eq. (48) and the equations derived in the previous section. In what follows, we discuss a design methodology of the two-stage LNA with those parameters. In NF calculation, the noise paprameter, $\gamma_{i}$ and $\delta_{i}$ of a 1-V LNA in $0.15-\mu \mathrm{m}$ FD-SOI CMOS technology based on the experimental results [11] are used.

The specifications of LNA are shown in Table 1. These are typical specifications for the LNA of a wireless local area network (WLAN) receiver.

(1) Gate lengths of $M_{1}$ and $M_{2}$

The gate length chosen is the minimum length of $0.14 \mu \mathrm{m}$ because NF decreases with scaling the gate length [5], [6].

(2) Input impedance

The input impedance of LNA, $R_{i n}^{\prime}$, is designed to be lower than $R_{s}$ in such a way that both the power gain and NF improve [4]. For simplicity, $R_{i n}^{\prime}$ is set to $R_{s}$.

(3) Bias currents of $M_{1}$ and $M_{2}$ 
Table 1 Specifications of LNA.

\begin{tabular}{|c|c|}
\hline Operation frequency & $5.4 \mathrm{GHz}$ \\
\hline$S_{11}$ & $<-10 \mathrm{~dB}$ \\
\hline Power Gain & $>20 \mathrm{~dB}$ \\
\hline NF & $<2.0 \mathrm{~dB}$ \\
\hline Current Consumption & $8.0 \mathrm{~mA}$ \\
\hline Supply Voltage & $1.0 \mathrm{~V}$ \\
\hline
\end{tabular}

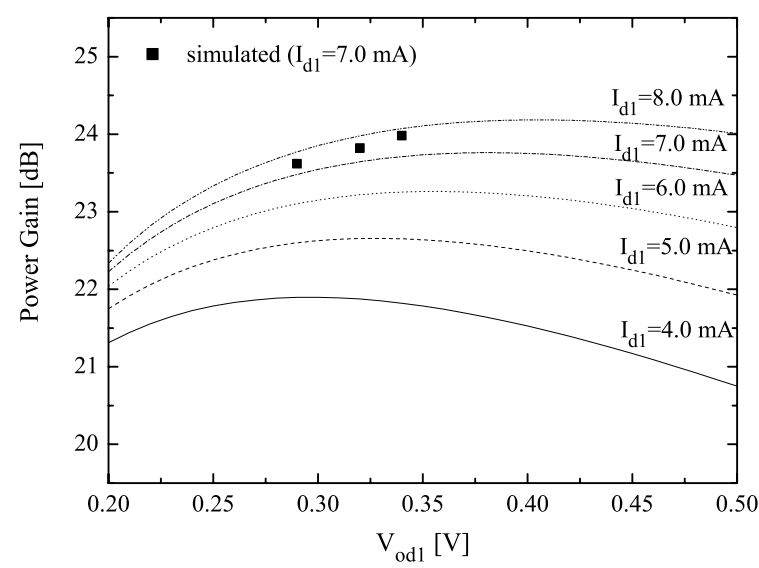

Fig. 7 Calculated and simulated power gain versus $V_{\text {od } 1}$.

Equation (20) shows that the common-gate stage has a small influence on the overall power gain. Therefore, the bais current of $M_{1}, I_{d 1}$, is chosen to satisfy the power gain specification.

Figure 7 shows the calculated power gain versus $V_{o d 1}$ as a parameter of $I_{d 1}$. Note that $g_{m 2}$ is set to infinity in Eq. (20). For comparison, simulated results $\left(I_{d 1}=7.0 \mathrm{~mA}\right)$ are also plotted in the figure. This and the following simulations of the LNA are carried out using the small-signal and noise FD-SOI MOS device model [11]. The calculated results are comparable to the simulated ones. The given specification is satisfied in the range of $4.0-8.0 \mathrm{~mA}$. Taking account of process and temperature variations, we choose $I_{d 1}$ to be $7.0 \mathrm{~mA}$ for a power gain of $23 \mathrm{~dB}$ including a $3 \mathrm{~dB}$ margin and $I_{d 2}=1.0 \mathrm{~mA}$ from the power specification.

\section{(4) Overdrive of $M_{1}$}

For NF and IIP3 of the input stage, $\overline{\left|i_{o, M_{2}}\right|^{2}}$ and $g_{m 2}$ are set to 0 and infinity in Eq. (32), respectively, and $A_{2, I I P_{3}}^{2}$ to infinity in Eq. (36). Figure 8 shows the calculated and simulated NF and $\operatorname{IIP}_{3}$ versus $V_{o d 1}$ at $I_{d 1}=7.0 \mathrm{~mA}$. Again, the calculated results are comparable to the simulated ones.

From the figure, the increase of $V_{o d 1}$ results in better noise performance of the LNA, but poor linearity. The degradation of linearity can be explained as follows: Under the impedance matching condition, the constant current $i_{\text {in }}=v_{s} / 2 R_{s}$ injects in the LNA. The small-signal voltage of gate-source of $M_{1}$ is given by

$$
v_{g s 1} \simeq \frac{i_{i n}}{j \omega_{0} C_{g s 1}} .
$$

The gate-source capacitance of $M_{1}, C_{g s 1}$, decreases with increasing $V_{o d 1}$ under constant $I_{d 1}$, resulting in the increase

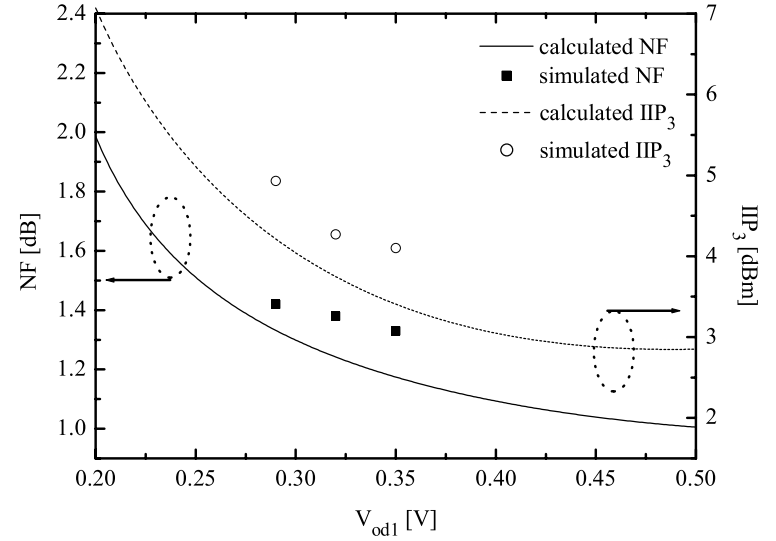

Fig. 8 Calculated and simulated $\mathrm{NF}$ and $\mathrm{IIP}_{3}$ versus $V_{o d 1}$.

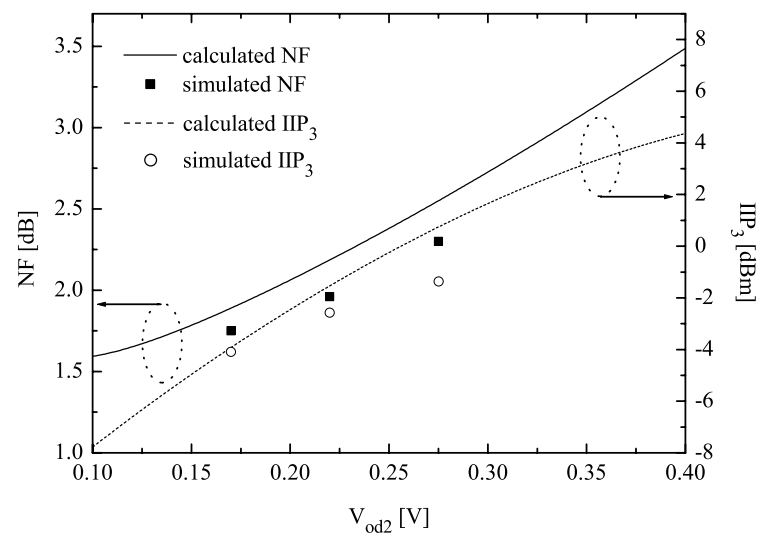

Fig. 9 Calculated and simulated $\mathrm{NF}$ and $\mathrm{IIP}_{3}$ versus $V_{\text {od } 2}$.

of $v_{g s}$. Although the MOSFET generally has higher linearity with increasing $V_{o d 1}$, in the range of $0.20-0.50 \mathrm{~V}$, the degradation of linearity due to the increase in $v_{g s 1}$ becomes significant, causing poor linearity. Therefore, a smaller $V_{o d 1}$ results in an LNA with higher linearity. Taking account of an additional noise of the output stage, we choose $V_{o d 1}=0.32 \mathrm{~V}$, resulting in $W_{1}=120 \mu \mathrm{m}$.

(5) Overdrive of $M_{2}$

Figure 9 shows the calculated and simulated NF and $\mathrm{IIP}_{3}$ versus $V_{o d 2}$ at $I_{d 1}=7.0 \mathrm{~mA}, V_{o d 1}=0.32 \mathrm{~V}$ and $I_{d 2}=1.0 \mathrm{~mA}$. The differences between the simulated and calculated results at a higher $V_{o d 2}$ originate form the simplified noise equivalent circuit including the Miller effect (Fig. 5) and the IIP $_{3}$ approximation of two non-linear stages in cascade (Eq. (36)).

Although the increase of $V_{o d 1}$ results in better noise performance and poor linearity as shown in Fig. 8, the increase of $V_{o d 2}$ leads to the opposite results: Better linearity and poor noise performance. This is because decreasing $g_{m 2}$ increases the Miller factor $M$, resulting in a higher NF. Therefore, an LNA with both the given noise performance and high linearity can be achieved by choosing the highest $V_{o d 2}$, that is, $0.17 \mathrm{~V}$ satisfying the NF specification, resulting in $W_{2}=50 \mu \mathrm{m}$.

(6) Inductors and capacitors 
Substituting the determined parameters into the equation of $L_{q}$ derived from Eqs. (2), (3), (11), (15) and (16), $L_{g}=3.3 \mathrm{nH}$ is derived and then $L_{s}=1.0 \mathrm{nH}$ from Eqs. (3) and (16).

Finally, the resonance condition gives inductors $L_{i}$ of $3.1 \mathrm{nH}$ and capacitors $C_{i}$ of $120 \mathrm{fF}(i=1,2,3)$. We used the ac-coupling capacitor $C_{c}$ of $3.3 \mathrm{pF}$ which is large enough not to affect RF signals.

From the above consideration, the following performances are expected: The power gain of $23 \mathrm{~dB}$, NF $1.8 \mathrm{~dB}$, $\mathrm{IIP}_{3}-4.1 \mathrm{dBm}$ and power consumption $8 \mathrm{~mW}$.

\section{Experimental Results}

A two-stage LNA is implemented in a $0.15-\mu \mathrm{m}$ FD-SOI CMOS process with five metal layers and metal-insulatormetal (MIM) capacitors. The cut-off frequency of a $0.15 \mu \mathrm{m}$ NMOS consisting of 48 fingers with a unit of $5 \mu \mathrm{m}$ width is about $54 \mathrm{GHz}$ at $V_{d s}=1 \mathrm{~V}$ and $I_{d}=7 \mathrm{~mA}$ [1]. The microphotograph of the fabricated LNA is shown in Fig. 10. All the pads are not ESD protected. A quality factor of on-chip inductors is about 11 at $5.4 \mathrm{GHz}$. The chip area is $0.78 \mathrm{~mm} \times 0.73 \mathrm{~mm}$. For the measurement, a buffer with a voltage gain of around $0 \mathrm{~dB}$ is implemented. The buffer has a small influence on the input reflection and noise performance of the LNA because the LNA has high reverse isolation and high gain. The current consumption of the LNA without the buffer is $8.3 \mathrm{~mA}$ at a $1-\mathrm{V}$ supply voltage.

$S$-parameters, NF and $\mathrm{IIP}_{3}$ of the LNA are measured using on-wafer RF probes. The above characteristics of the LNA with an ideal inductor $L_{q}$ in series to the gate are calculated based on the measured data [11]. This avoids instrumental error originating from bonding wires.

Figure 11 shows measured and simulated $S_{11}$ and $S_{21}$, i.e., power gain of the LNA. The impedance matching is achieved $\left(S_{11}<-10 \mathrm{~dB}\right)$ around $5.4 \mathrm{GHz}$ where the power gain is $23 \mathrm{~dB}$. Figure 12 shows measured and simulated NF versus frequency. An NF of $1.7 \mathrm{~dB}$ is obtained at $5.4 \mathrm{GHz}$. The measured results agree well with those simulated by the the small-signal and noise model [11].

Figure 13 shows the measured output power of fun-

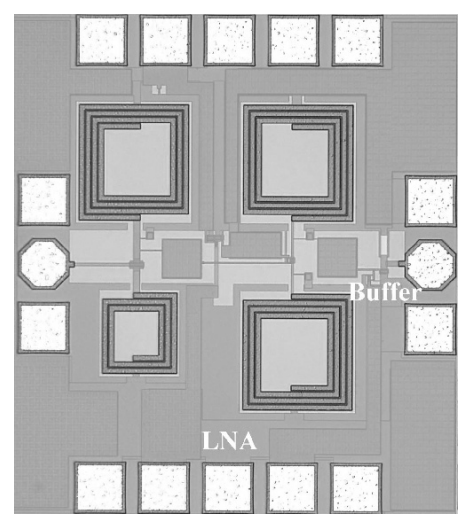

Fig. 10 Microphotograph of LNA. damental tone and third-order intermodulation (IM3) products for two tones $(5.4$ and $5.41 \mathrm{GHz})$. The measured $\mathrm{IIP}_{3}$ is about $-18.0 \mathrm{dBm}$. This result includes the nonlinearity of the buffer, which can be eliminated by using the follow equation:

$$
\frac{1}{A_{L N A, I I P_{3}}^{2}} \simeq \frac{1}{A_{L N A+B u f, I I P_{3}}^{2}}-\frac{A_{v, L N A}^{2}}{A_{B u f, I I P_{3}}^{2}}
$$

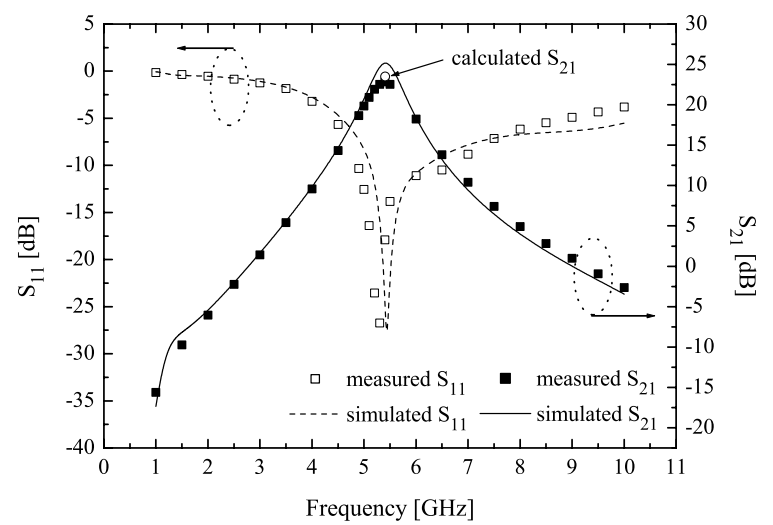

Fig. 11 Measured and simulated $S_{11}$ and $S_{21}$ of LNA.

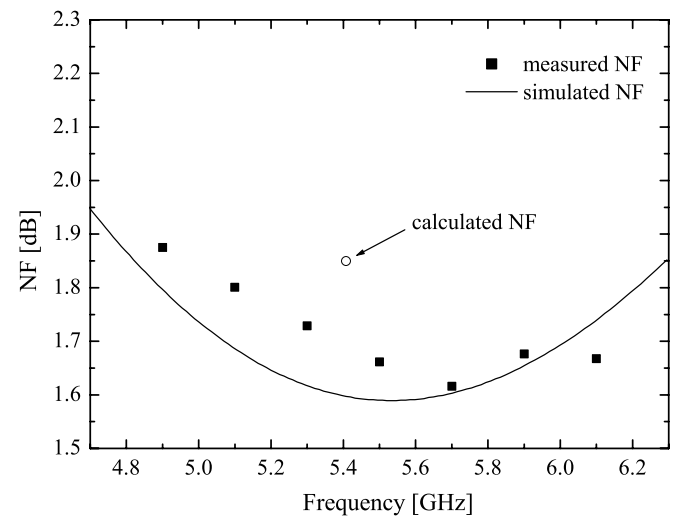

Fig. 12 Measured and simulated NF of LNA

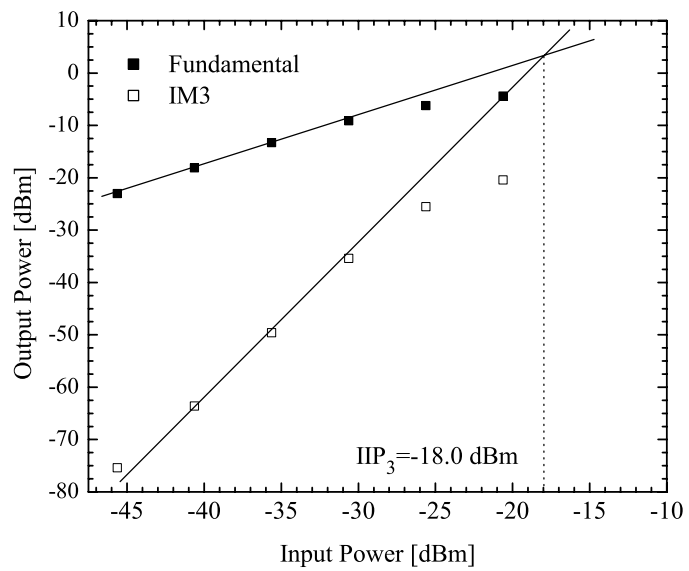

Fig. 13 Measured $\mathrm{IIP}_{3}$ of LNA including buffer. 
Table 2 Comparison of performances of LNA.

\begin{tabular}{|c|c|c|c|c|}
\hline & Specification & Measurement & Calculation & Simulation \\
\hline Power Gain @ 5.4 GHz & $20 \mathrm{~dB}$ & $23 \mathrm{~dB}$ & $23 \mathrm{~dB}$ & $25 \mathrm{~dB}$ \\
\hline NF @ 5.4 GHz & $2.0 \mathrm{~dB}$ & $1.7 \mathrm{~dB}$ & $1.8 \mathrm{~dB}$ & $1.6 \mathrm{~dB}$ \\
\hline $\mathrm{IIP}_{3}$ & N/A & $-6.1 \mathrm{dBm}$ & $-4.1 \mathrm{dBm}$ & $-3.0 \mathrm{dBm}$ \\
\hline
\end{tabular}

Table 3 Measured performance of two LNAs.

\begin{tabular}{|c|c|c|}
\hline & $L_{s}=1.0 \mathrm{nH}$ & $L_{s}=0.8 \mathrm{nH}$ \\
\hline$S_{11}$ & \multicolumn{2}{|c|}{$<-10 \mathrm{~dB}$} \\
\hline Power Gain @ 5.4 GHz & $23 \mathrm{~dB}$ & $26 \mathrm{~dB}$ \\
\hline NF @ 5.4 GHz & $1.7 \mathrm{~dB}$ & $1.4 \mathrm{~dB}$ \\
\hline IIP $_{3}$ & $-6.1 \mathrm{dBm}$ & $-7.0 \mathrm{dBm}$ \\
\hline Current Consumption & \multicolumn{2}{|c|}{$8.3 \mathrm{~mA}$} \\
\hline Supply Voltage & \multicolumn{2}{|c|}{$1.0 \mathrm{~V}$} \\
\hline
\end{tabular}

where $A_{v, L N A}$ is the voltage gain of the LNA, $A_{L N A, I I P_{3}}$, $A_{B u f, I I P_{3}}$ and $A_{L N A+B u f, I I P_{3}}$ represent the $\mathrm{IIP}_{3}$ of the LNA, buffer and LNA including the buffer in the expression of voltage amplitude, respectively. Substituting measured $\mathrm{IIP}_{3, \mathrm{LNA}+\mathrm{Buf}}=-18.0 \mathrm{dBm}, \mathrm{IIP}_{3, \mathrm{Buf}}=10.8 \mathrm{dBm}$ and $A_{v, L N A}$ $=22.5 \mathrm{~dB}$ into Eq. (50), the $\mathrm{IIP}_{3}$ of the LNA is achieved about $-6.1 \mathrm{dBm}$.

Among other characteristics, $S_{12}$ and $S_{22}$ are below $-42 \mathrm{~dB}$ and $-10 \mathrm{~dB}$, respectively.

Table 2 shows a comparison of performances of the LNA. The simulated and calculated power gain and NF are consistent with the measured results and the fabricated LNA, thus satisfying the specifications. The calculated $\mathrm{IIP}_{3}$ agrees with the simulated one, but these results are slightly different from the measured $\mathrm{IIP}_{3}$. The difference is due to inaccurate FD-SOI MOS device parameters used in the simulation and calculation.

Table 3 shows a performance summary of the above LNA $\left(L_{s}=1.0 \mathrm{nH}\right)$ and other fabricated LNA with $L_{s}=$ $0.8 \mathrm{nH}$. The table suggests that power gain and noise performance of the LNA can be improved by lowering $L_{s}$.

\section{Conclusion}

We investigated high frequency characteristics of a lowvoltage two-stage CMOS LNA. Based on the derived equations of power gain, $\mathrm{NF}$ and $\mathrm{IIP}_{3}$, we proposed a new design methodology of the LNA. By using the overdrive voltages of the MOSFETs as adjustable parameters, we demonstrated systematic design of an LNA under given specifications. A $1-\mathrm{V}$ 5.4-GHz LNA was realized in $0.15-\mu \mathrm{m}$ FD-SOI CMOS technology. The LNA achieved a power gain of $23 \mathrm{~dB}, \mathrm{NF}$ of $1.7 \mathrm{~dB}$ and $\mathrm{IIP}_{3}$ of $-6.1 \mathrm{dBm}$ with a power consumption of $8.3 \mathrm{~mW}$. The results are consistent with the calculated results obtained from the derived equations. This ensures the validity of the equations and proposed design methodology.

\section{Acknowledgements}

This study is supported by Semiconductor Technology Academic Research Center (STARC). The chip design in this work is also supported by VLSI Design and Education Cen- ter (VDEC), the University of Tokyo in collaboration with Synopsys, Inc., Cadence Design System, Inc. and Agilent Technologies Japan, Ltd.

\section{References}

[1] F. Ichikawa, Y. Nagatomo, Y. Katakura, M. Itoh, S. Itoh, H. Matsuhashi, T. Ichimori, N. Hirashita, and S. Baba, "Fully depleted SOI process and technology for digital and RF applications," SolidState Electron., vol.48, pp.999-1006, 2004.

[2] A.O. Adan, T. Yoshimatsu, S. Shitara, N. Tanba, and M. Fukumi, "Linearity and low-noise performance of SOI MOSFETs for RF applications," IEEE Trans. Electron Devices, vol.49, no.5, pp.881888, May 2002.

[3] M. Brandolini, P. Rossi, D. Manstretta, and F. Svelto, "Toward multistandard mobile terminals-fully integrated receivers requirements and architecures," IEEE Trans. Microw. Theory Tech., vol.53, no.3, pp.1026-1038, March 2005.

[4] J. Janssens and M. Steyaert, CMOS Cellular Receiver Front-Ends: From Specification to Realization, Kluwer Academic Publishers, Boston, 2002.

[5] D.K. Shaeffer and T.H. Lee, "A 1.5-V, 1.5-GHz CMOS low noise amplifier," IEEE J. Solid-State Circuits, vol.32, no.5, pp.745-759, May 1997.

[6] D.K. Shaeffer and T.H. Lee, "Corrections to "A 1.5-V, 1.5-GHz CMOS low noise amplifier,"” IEEE J. Solid-State Circuits, vol.40, no.6, pp.1397-1398, June 2005.

[7] T. Kihara, G. Kim, Y. Shimizu, B. Murakami, K. Ueda, M. Goto, T. Matsuoka, and K. Taniguchi, "Design of CMOS low-noise amplifier considering noise and linearity," IEICE Trans. Electron. (Japanese Edition), vol.J89-C, no.2, pp.72-75, Feb. 2006.

[8] C.L. Hsiao, R.M. Weng, K.Y. Lin, and H.C. Wei, "A sub 1 V 2.4 GHz CMOS variable-gain low noise amplifier," IEICE Trans. Electron., vol.E87-C, no.6, pp.1003-1004, June 2004.

[9] B. Razavi, RF Microelectronics, Prentice Hall PTR, New Jersey, 1998.

[10] B. Razavi, Design of Analog CMOS Integrated Circuits, McGrawHill, New York, 2001.

[11] G. Kim, B. Murakami, M. Goto, T. Kihara, K. Nakamura, Y. Shimizu, T. Matsuoka, and K. Taniguchi, "Small-signal and noise model of FD-SOI MOS devices for low noise amplifier," Jpn. J. Appl. Phys., Pt.1, vol.45, no.9A, pp.6872-6877, Sept. 2006.

\section{Appendix: Derivations of Output Noise Originating from $M_{1}$ and $M_{2}$}

The induced-gate noise correlates to the drain noise. Considering the correlation between the gate and drain noises, the induced-gate noise is expressed as the sum of correlated and uncorrelated components:

$$
\begin{aligned}
\overline{\left|i_{n g}\right|^{2}} & =\overline{\left|i_{\text {ngc }}\right|^{2}}+\overline{\left|i_{\text {ngu }}\right|^{2}} \\
& =\overline{\left|i_{n g}\right|^{2}}|c|^{2}+\overline{\left|i_{n g}\right|^{2}}\left(1-|c|^{2}\right)
\end{aligned}
$$

where $i_{n g}$ is given by Eq. (23). The correlation coefficient, $c$, between the induced-gate and drain noise currents is given 
by [5], [6]

$$
c=\frac{\overline{i_{n g} \cdot i_{n d}^{*}}}{\sqrt{\overline{\left|i_{n g}\right|^{2}}} \sqrt{\overline{\left|i_{n d}\right|^{2}}}} .
$$

From the correlation shown above, the output noise due to $M_{i}$ is expressed as

$$
\begin{aligned}
\overline{\left|i_{o, M_{i}}\right|^{2}=} & \overline{\left|i_{o, n d i}+i_{o, n g i}\right|^{2}} \\
= & \overline{\left|i_{o, n d i}+i_{o, n g c i}\right|^{2}}+\overline{\left|i_{o, n g u i}\right|^{2}} \\
= & \overline{\left|i_{o, n d i}\right|^{2}}+\overline{i_{o, n g c i} \cdot i_{o, n d i}^{*}}+\overline{i_{o, n d i} \cdot i_{o, n g c i}^{*}} \\
& \quad+\overline{\left|i_{o, n g c i}\right|^{2}}+\overline{\left|i_{o, \text { ngui }}\right|^{2}},
\end{aligned}
$$

where $i_{o, n d i}, i_{o, n g c i}$ and $i_{o, n g u i}$ are the output noise currents originating from $i_{n d i}, i_{n g c i}$ and $i_{n g u i}$, respectively.

From Fig. 5, the transfer function from the drain noise current $i_{n d 1}$ to the output $i_{o, n d 1}$ is given by

$$
H_{n d 1}\left(j \omega_{0}\right)=\frac{R_{e q}+r_{n q s, e f f}}{\left(R_{e q}+R_{i n}\right)\left(1+1 / g_{m 2} R_{I}\right)} .
$$

The transfer function from the induced-gate noise current $i_{n g 1}$ to the output $i_{o, n g 1}$ is also given by

$$
H_{n g 1}\left(j \omega_{0}\right)=\frac{\omega_{T 1}\left(R_{e q}+j / \omega_{0} C_{g s 1, e f f}\right)}{j \omega_{0}\left(1+\alpha_{g d} M\right)\left(R_{e q}+R_{i n}\right)\left(1+1 / g_{m 2} R_{I}\right)} .
$$

Using Eqs. (22), (23), (A·4) and (A· 5), we have

$$
\begin{aligned}
& \overline{\left|i_{o, n d 1}\right|^{2}}=\left|H_{n d 1}\left(j \omega_{0}\right)\right|^{2} \overline{\left|i_{n d 1}\right|^{2}} \\
& =\frac{4 k_{B} T \gamma_{1} g_{m 1}\left(R_{e q}+r_{n q s, e f f}\right)^{2} \Delta f}{\alpha_{1}\left(R_{e q}+R_{i n}\right)^{2}\left(1+1 / g_{m 2} R_{I}\right)^{2}} \\
& \overline{i_{o, n g c 1} \cdot i_{o, n d 1}^{*}}+\overline{i_{o, n d 1} \cdot i_{o, n g c 1}^{*}} \\
& =H_{n g c 1}\left(j \omega_{0}\right) i_{n g c 1} \cdot H_{n d 1}^{*}\left(j \omega_{0}\right) i_{n d 1}^{*} \\
& \quad+H_{n d 1}\left(j \omega_{0}\right) i_{n d 1} \cdot H_{n g c 1}^{*}\left(j \omega_{0}\right) i_{n g c 1}^{*} \\
& =-2|c| \sqrt{\frac{\delta_{1}}{\kappa_{1} \gamma_{1}}} \frac{4 k_{B} T \gamma_{1} g_{m 1} R_{e q}\left(R_{e q}+r_{n q s, e f f}\right) \Delta f}{\left(1+\alpha_{g d} M\right)\left(R_{e q}+R_{i n}\right)^{2}\left(1+1 / g_{m 2} R_{I}\right)^{2}}, \\
& \overline{\left|i_{o, n g c 1}\right|^{2}}+\overline{\left|i_{o, n g u 1}\right|^{2}} \\
& =\left|H_{n g 1}\left(j \omega_{0}\right)\right|^{2} \overline{\left|i_{n g 1}\right|^{2}} \\
& =\frac{4 k_{B} T \alpha_{1} \delta_{1} g_{m 1}\left(R_{e q}^{2}+1 / \omega_{0}^{2} C_{g s 1, e f f}^{2}\right) \Delta f}{\kappa_{1}\left(1+\alpha_{g d} M\right)^{2}\left(R_{e q}+R_{n}\right)^{2}\left(1+1 / g_{m 2} R_{I}\right)^{2}} .
\end{aligned}
$$

Substituting Eqs. (A.6)-(A. 8) into Eq. (A.3), Eq. (25) can be derived.

In the same way, the output noise originating from $M_{2}$ is also derived. From Fig. 6, the transfer functions from the drain and induced-gate noise currents to the output are derived as

$$
\begin{aligned}
& H_{n d 2}\left(j \omega_{0}\right)=\frac{1}{1+g_{m 2} R_{I}}, \\
& H_{n g 2}\left(j \omega_{0}\right)=\frac{1}{1+1 / g_{m 2} R_{I}} .
\end{aligned}
$$

From Eqs. (22), (23), (A. 9) and (A. 10), we have

$$
\begin{aligned}
& \overline{\left|i_{o, n d 2}\right|^{2}}=\frac{4 k_{B} T \gamma_{2} g_{m 2}}{\alpha_{2}\left(1+g_{m 2} R_{I}\right)^{2}}, \\
& \overline{i_{o, n g c 2} \cdot i_{o, n d 1}^{*}}+\overline{i_{o, n d 2} \cdot i_{o, n g c 2}^{*}}=0, \\
& \overline{\left|i_{o, n g c 2}\right|^{2}}+\overline{\left|i_{o, n g u 2}\right|^{2}}=\frac{4 k_{B} T \delta_{2} \alpha_{2}\left(\omega_{0} C_{g s 2}\right)^{2}}{\kappa_{2} g_{m 2}\left(1+1 / g_{m 2} R_{I}\right)^{2}} .
\end{aligned}
$$

Substituting Eqs. (A.11)-(A.13) into Eq. (A.3), Eq. (28) can be derived.

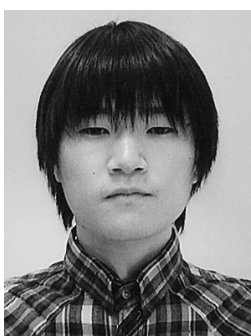

Takao Kihara received the B.S. and M.S degrees in electronic engineering from Osaka University, Osaka, Japan, in 2005 and 2006, respectively. He is now working towards his $\mathrm{Ph} . \mathrm{D}$. degree at Osaka University. His current research interests include CMOS RF circuits.

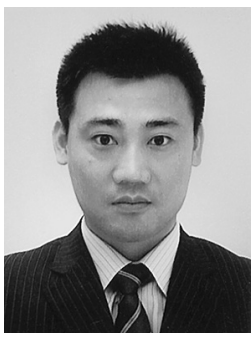

Guechol Kim recevied the B.S. and M.S degrees in electronic engineering from Mokpo National University, Mokpo, Korea, in 2000 and 2003 , respectively. He is now working towards his Ph.D. degree at Osaka University. His current research interests include FD-SOI MOSFET and RF circuits.

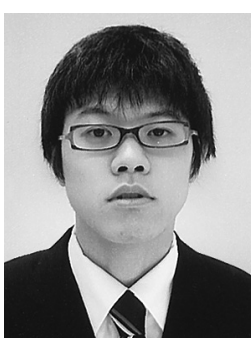

Masaru Goto received the B.S. degree in electronic engineering from Osaka University, Osaka, Japan, in 2005. He is now working towards his M.S degree at Osaka University. His current research interests include CMOS RF circuits. 


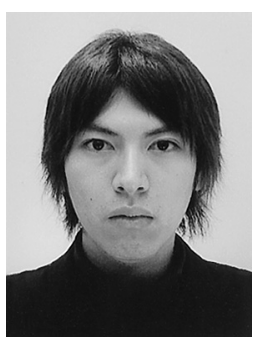

Keiji Nakamura received the B.S. degree in electronic engineering from Osaka University, Osaka, Japan, in 2006. He is now working towards his M.S degree at Osaka University. His current research interests include CMOS RF circuits and phase locked loops.

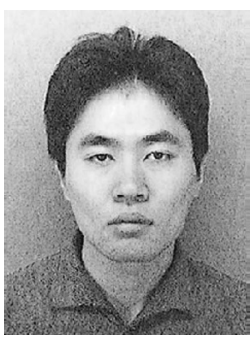

Yoshiyuki Shimizu received the B.S. M.S. and Ph.D. degrees in electronic engineering from Osaka University, Osaka, Japan, in 2000, 2002 and 2005 respectively. He is now a postdoctoral fellow at Osaka University.

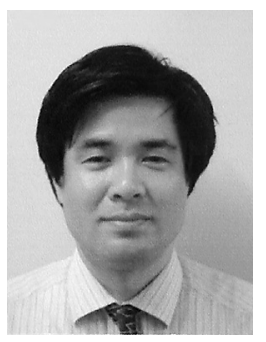

Toshimasa Matsuoka received the B.S., M.S. and Ph.D. degrees in electronic engineering from Osaka University, Osaka, Japan, in 1989, 1991 and 1996, respectively. During 1991-1998, he worked for the Central Research Laboratories, Sharp Corporation, Nara, Japan, where he was engaged in the research and development of deep submicron CMOS devices and ultra thin gate oxides. Since 1999, he has been working for Osaka University, where he is Associate Professor now. His current research includes MOS device modeling and CMOS RF circuits. Dr. Matsuoka is a member of the Japan Society of Applied Physics, the IEEJ, and the IEEE.

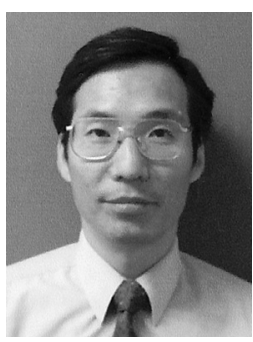

Kenji Taniguchi received the B.S., M.S. and Ph.D. degrees from Osaka University, Osaka, Japan, in 1971, 1973 and 1986, respectively. From 1973 to 1986, he worked for Toshiba Research and Development Center, Kawasaki, Japan where he was engaged in process modeling and the design of MOS LSI fabrication technology. He was a Visiting Scientist at Massachusetts Institute of Technology, Cambridge, from July 1982 to November 1983. Presently, he is a Professor of Electrical, Electronic and Information Engineering at Osaka University. His current research interests are analog circuits, radio frequency circuits, device physics and process technology. Prof. Taniguchi is a member of the Japan Society of Applied Physics. He is a fellow of the IEEE. 